\title{
Evaluation of Mobile Electronic Devices in Detecting Optic Disc and Visual Field Parameters in Patients with Glaucoma at a Tertiary Hospital in Northeastern Tanzania
}

\author{
Francisco Alcides Mulobuana1*, Tolbert Sonda², Martin M. Rwabilimbo ${ }^{3}$, William Makupa4 \\ ${ }^{1}$ Kilimanjaro Christian Medical University College, Moshi, Tanzania \\ ${ }^{2}$ Kilimanjaro Clinical Research Institute, Moshi, Tanzania \\ ${ }^{3}$ Department of Epidemiology and Applied Biostatistics, Kilimanjaro Christian Medical College University, Moshi, Tanzania \\ ${ }^{4}$ Kilimanjaro Christian Medical Centre, Moshi, Tanzania \\ Email: ^franciscofranciscoalcides@gmail.com, makupauw@yahoo.com, t.sonda@kcri.ac.tz,martinmujun@gmail.com
}

How to cite this paper: Mulobuana, F.A., Sonda, T., Rwabilimbo, M.M. and Makupa, W. (2020) Evaluation of Mobile Electronic Devices in Detecting Optic Disc and Visual Field Parameters in Patients with Glaucoma at a Tertiary Hospital in Northeastern Tanzania. Open Journal of Ophthalmology, 10, 220-228.

https://doi.org/10.4236/ojoph.2020.103024

Received: May 23, 2020

Accepted: August 17, 2020

Published: August 20, 2020

Copyright $\odot 2020$ by author(s) and Scientific Research Publishing Inc. This work is licensed under the Creative Commons Attribution International License (CC BY 4.0).

http://creativecommons.org/licenses/by/4.0/ (c) (i) Open Access

\begin{abstract}
Background: Glaucoma is a serious public health problem since it causes visual impairment impacting social, mental and physical health of an individual. Diagnosis and management of glaucoma continue to be a challenge due to few qualified personnel and high cost of the equipment. The use of portable Eye Examination Kit such as Smartphone and tablets can be used in glaucoma screening for taking high-resolution fundus photos for optic disc and visual field parameters, respectively. This study was conducted to evaluate the applicability of mobile electronic device to detect optic disc and visual field parameters for glaucoma in a resource limited setting. Objective: To evaluate the applicability of mobile electronic devices to detect optic disc and visual field parameters for glaucoma. Methodology: Across-sectional study was conducted at KCMC Eye department from October 2018 to June 2019. Study included 140 participants attending eye clinic of which 67 had glaucoma and 73 without glaucoma. Clinical and socio-demographic data were collected using a structured questionnaire and analysed using Stata 15. Glaucoma examination was made on the right eye and photo comparison made between those with and without glaucoma. PEEK Smartphone fundus photo examination was compared with the gold standard machine (Slit Lamp Biomicroscopy), Amsler grid chart installed on a tablet (Microsoft surface, internal storage 256 GB, 2013) and contrast sensitivity compared with Humphrey field analyser (i series, model 740117434, Carl Zeiss Meditec). Results: The kappa (k) agreement between Slit lump biomicroscopy fundus view image and PEEK Smartphone concerning the optic disc measurement was 0.92 with sensitivity and specificity
\end{abstract}


of $90.32 \%$ (95\% CI: 80.12 - 96.37) and 93.59\% (95\% CI: 85.67 - 97.89) with a p $<0.001$ respectively. An agreement between Amsler grid and Humphrey Field Analyser was 0.67 with sensitivity of $33.33 \%$ (95\% CI: 20.76 - 47.92) and specificity of $86.52 \%$ (95\% CI: $77.63-92.83)$ with a $p<0.005$. An agreement for contrast sensitivity and Humphrey Field Analyser was 0.51 with sensitivity of $48.91 \%$ (95\% CI: 38.3 - 59.56) and specificity of $54.17 \%$ (95\% CI: 39.17 - 68.63) and $\mathrm{p}$ value of 0.729 . Conclusion: PEEK Smartphone fundus image specificity was almost in perfect agreement compared with Amsler grid and Contrast sensitivity. The PEEK Smartphone fundus view optic disc parameter for vertical cup to disc ratio has a potential to enhance detection of glaucoma and thus improve its management in resource-limited settings.

\section{Keywords}

Glaucoma, PEEK Smartphone, Contrast Sensitivity, Amsler Grid, Kilimanjaro

\section{Introduction}

Glaucoma is a serious public health problem causing visual impairment, which subsequently impacts social, mental and physical health of an individual. The number of people visually impaired around the world is estimated to be over 200 million; of whom more than 30 million are blind; amongst these, more than $80 \%$ are people at middle age and more [1]. Glaucoma is one of the leading causes of blindness and the prevalence in Africa is about $15 \%$ with some countries such as Western Cameroon with 8.2\%, and those aged 40 years and more were $5.0 \%$ in Nigeria and $8.5 \%$ in Ghana and in Kenya, according to the same report the prevalence was $4.3 \%$ for those aged 50 years and more [2]. In Tanzania having prevalence of all types of glaucoma is $4.16 \%$ and the primary open angle glaucoma as the main type [3].

Early diagnosis for effective detection in high risk populations is crucial to prevent loss of vision related with glaucoma [4]. The gold standard for diagnosing glaucoma is perimetry such as Humphrey field device, but by the time the patients come at the health facilities and these defects detected, the visual loss is already installed and permanent [5]. New technological development of portable devices for glaucoma screening such as Smartphone or tablets is worth exploring. The advantages of these tools are related with their accessibility around the world; convenience, low cost compared with gold standard devices, decreased travel time of the patients to the medical clinics away from their communities for follow up, increased access to specialized care for glaucoma, and decreased patient costs [4]. Smartphones or tablets are handy and can be used for taking high-resolution fundus photos image and help in diagnosing and implement early treatment in patients with glaucoma and thus avoiding blindness in remote or under-serviced communities such as rural or remote areas where there are limited ocular specialists [4]. 
This study was conducted to evaluate the applicability of mobile electronic devices to detect optic disc and visual field parameters for glaucoma.

\section{Materials and Methods}

\subsection{Study Design and Area}

This was an analytical cross-sectional study for detection of glaucoma; conducted at KCMC Eye Clinic during October 2018 to June 2019. KCMC is a tertiary referral hospital for over 15 million people, which is located in the foothills of Mount Kilimanjaro in Northern Tanzania. The eye department attends more than two thousand patients a year, with an admission service which contains 62 beds and many types of surgery is done in this setting such as glaucoma surgery (e.g. trabeculectomies, goniotomies), ocular plastic surgery, corneal transplant surgery, retinal surgery and many others.

\subsection{Ethical Considerations}

Ethical approval for this study has been obtained from the KCMU Co Ethical Committee prior to the commencement of this study with certificate number 2321. Permission to conduct this study has been sought from the Director of the hospital and Head of Eye Department. A written informed consent was obtained from each participant or from parents or relatives before enrolment into the study. Confidentiality and privacy of study subjects was maintained by the use of unique identifiers and only the research team had access to data.

\subsection{Ophthalmological Procedure}

Patients attending Eye clinic at KCMC hospital were conveniently sampled and enrolled into this study and a sample size of adequate sensitivity and a power of 95\% confidence interval with the precision of estimate which does not exceed from $7 \%$ were used for calculation [6].

The inclusion criteria was patients above 18 years who consent to participate in the research study, patients with and without glaucoma, patients with refractive error less than +4.75 dioptres and less than -6.0 dioptres and the exclusion criteria was patients with blindness $\mathrm{BE}$ and other ocular cormobilities.

All enrolled patients underwent a medical history, visual acuity by Snellen chart recorded in logMAR, portable Eye examination Kit (PEEK) visual acuity (in $\log \mathrm{MAR}$ ) and contrast sensitivity by Smartphone (SonyExperia, m1, d2403, 2015), Auto refractor and Amsler grid tested on the tablet (Microsoft surface, internal storage 256 GB, 2013) and visual field by Humphrey field (Humphrey field analyzer i series, model 740117434, Carl Zeiss Meditec) was done by a trained personnel who was blinded for glaucoma. After all examination, those participants with and without glaucoma were selected if the vertical cup to disc ratio (VCDR) as less than 0.7 and equal or more than 0.7 , visual field within normal limits and outside normal limits, respectively. Also patients with refractive error less than +4.75 diopters and less than -6.0 diopters for spherical and 
cylindrical were included. For contrast sensitivity, those with equal or less than $2.5 \%$ were considered normal and those with more than $2.5 \%$ were considered abnormal and the comparison was made with visual field from Humphrey Field Analyser. Amsler grid measured the areas of scotoma, those with no areas of scotoma were considered normal and those with areas of scotoma were considered abnormal and comparison with visual field results 10 - 2 from Humphrey field was made and recorded [7] [8].

\subsection{Statistical Analysis}

All analyses were performed using Stata 15. Descriptive statistics were determined for all demographic and ocular characteristics and presented as proportions. Data for the right eye was chosen for analyses. To compare Smartphone performance with the gold standard tools kappa coefficient of agreement was calculated. We also calculated sensitivity, specificity, positive and negative predictive value of Smartphone in detecting glaucoma.

\section{Results}

\subsection{Socio-Demographic and Clinical Characteristics of Study Participants}

A total 140 patients who met the inclusion criteria were included in the analysis (Table 1). Out of 140 participants 74 (52.9\%) aged 18 - 50 with 74 (52.9\%) being female. It was observed that those with normal visual acuity measured by Snellen VA were more predominant $128(91.4 \%)$ with the similar results for PEEK visual acuity 129 (92.1\%). Refractive error for spherical and cylindrical were evaluated and those scored $<+2.00$ diopters and those with $<-1.5$ diopters were $63(45 \%)$ and 96 (68.6\%), respectively.

Intraocular pressure was measured in all participants and found that most of the participants scored normal values in between 9 to $21 \mathrm{mmHg}, 1(0.7 \%)$ participants with less than $9 \mathrm{mmHg}$ and $4(2.9 \%)$ with more than $21 \mathrm{mmHg}$. For contrast sensitivity $92(65.71 \%)$ of the participants scored more than $2.5 \%$ and those with no scotoma for Amsler grid were 89 (63.6\%). For Amsler grid and Humphrey field severity was $89(63.6 \%)$ and 11 (79.3\%) for those with no scotoma, respectively. Participants with less than 0.7 vertical cup to disc ratio were $79(56.4 \%)$ as measured by Smartphone which was similar with the slit lamp measurement of vertical cup to disc ratio in 78 (55.7\%) participants.

According to Humphrey field, participants with within normal limits were 73 (52.14\%) compared with those with outside normal limits that were 67 (47.86\%). For mean deviation for those with $\leq 2 \mathrm{~dB}$ was 91 (65\%).

\subsection{Comparison between PEEK Smartphone Contrast Sensitivity and Humphrey Visual Field Analyser}

The agreement $(\mathrm{k})$ between PEEK Smartphone contrast sensitivity and Humphrey visual field analyser was 0.51 with $\mathrm{p}>0.05$ and sensitivity of $48.9 \%$ (95\% 
Table 1. Socio-Demographic and clinical characteristics of study participants $(\mathrm{N}=140)$.

\begin{tabular}{|c|c|c|c|}
\hline & Characteristics & $\mathrm{N}$ & $\%$ \\
\hline \multirow[t]{2}{*}{ Age } & $18-50$ & 74 & 52.86 \\
\hline & Above 50 & 66 & 47.14 \\
\hline \multirow[t]{2}{*}{ Sex } & Female & 74 & 52.86 \\
\hline & Male & 66 & 47.14 \\
\hline \multirow[t]{2}{*}{ Snellen VA } & $\leq 0.48 \operatorname{LogMAR}$ & 128 & 91.43 \\
\hline & $>0.48$ LogMAR & 12 & 8.57 \\
\hline \multirow{2}{*}{ PEEK VA Smartphone } & $\leq 0.48 \log M A R$ & 129 & 92.14 \\
\hline & $>0.48$ LogMAR & 11 & 7.86 \\
\hline \multirow[t]{5}{*}{ Refractive error spherical } & $<+2.00$ diopters & 63 & 45 \\
\hline & +2 to +4.75 diopters & 4 & 2.9 \\
\hline & $\leq 1.5$ diopters & 40 & 28.6 \\
\hline & -1.5 to -6.0 & 3 & 2.1 \\
\hline & Error & 30 & 21.4 \\
\hline \multirow[t]{5}{*}{ Refractive error cylindrical } & $<+2.00$ diopters & 9 & 6.4 \\
\hline & +2 to +4.75 diopters & 1 & 0.7 \\
\hline & $\leq 1.5$ diopters & 96 & 68.6 \\
\hline & -1.5 to -6.0 & 8 & 5.7 \\
\hline & Error & 26 & 18.6 \\
\hline \multirow[t]{3}{*}{ IOP } & $<9 \mathrm{mmhg}$ & 1 & 0.71 \\
\hline & 9 - $21 \mathrm{mmhg}$ & 135 & 96.43 \\
\hline & $>21 \mathrm{mmhg}$ & 4 & 2.86 \\
\hline \multirow[t]{2}{*}{ PEEK Smartphone Contrast sensitivity } & $\leq 2.5 \%$ & 48 & 34.28 \\
\hline & $>2.5 \%$ & 92 & 65.71 \\
\hline \multirow[t]{2}{*}{ PEEK Smartphone Amsler grid } & no scotoma & 89 & 63.57 \\
\hline & 1 or more scotoma & 51 & 36.43 \\
\hline \multirow[t]{3}{*}{ PEEK Smartphone Amsler grid severity } & Mild - no scotoma & 89 & 63.6 \\
\hline & Moderate - one scotona in one quadrant & 12 & 8.6 \\
\hline & Severe - scotoma in more than one quadrant & 39 & 27.9 \\
\hline \multirow[t]{2}{*}{ PEEK retina smartphone } & $\geq 0.7 \mathrm{VCDR}$ & 61 & 43.6 \\
\hline & $<0.7 \mathrm{VCDR}$ & 79 & 56.4 \\
\hline \multirow[t]{2}{*}{ Slit lamp biomicroscopy } & $\geq 0.7 \mathrm{VCDR}$ & 62 & 44.29 \\
\hline & $<0.7 \mathrm{VCDR}$ & 78 & 55.71 \\
\hline \multirow[t]{2}{*}{ Humphrey visual fieldanalyzer } & Outside normal limits is abnormal & 67 & 47.86 \\
\hline & Within normal limits is normal & 73 & 52.14 \\
\hline \multirow[t]{3}{*}{ Humphrey visual field analyzer $10-2$ severity } & Mild - no scotoma & 111 & 79.3 \\
\hline & Moderate - one scotona in one quadrant & 1 & 0.7 \\
\hline & Severe - scotoma in more than one quadrant & 28 & 20 \\
\hline \multirow[t]{2}{*}{ mean deviation } & $\leq 2 \mathrm{~dB}$ abnormal & 91 & 65.00 \\
\hline & $\geq 2 \mathrm{~dB}$ normal & 49 & 35.00 \\
\hline
\end{tabular}


CI: 38.34 - 59.56), specificity of 54.2\% (95\% CI: $39.17 \%-68.63 \%$ ), positive of 67.2\% (95\% CI: 54.60 - 78.15) and negative predictive value of $35.6 \%$ (95\% CI: 24.75 - 47.69) between contrast sensitivity Humphrey visual field analyser (Table 2).

\subsection{Comparison between PEEK Smartphone Amsler Grid and Humphrey Visual Field Analyser}

The agreement $(\mathrm{k})$ between PEEK Smartphone Amsler grid and Humphrey Visual Field Analyser was 0.67 with sensitivity and specificity were $33.3 \%$ (95\% CI: 20.76 - 47.92) and 86.5\% (95\% CI: 77.63 - 92.83), respectively. Positive and negative predictive values were 58.6\% (95\% CI: $38.94-76.48)$ and 69.4\% (95\% CI: 59.91 - 77.77), respectively and p < 0.005 (Table 3).

\subsection{Comparison between PEEK Retina Smartphone and Slit Lamp Biomicroscopy}

The agreement $(\mathrm{k})$ between Smartphone and Slit lamp biomicroscopy was 0.92 with sensitivity and specificity of $90.3 \%$ (95\% CI: 80.12 - 96.37) and 93.6\% (95\% CI: 85.67 - 97.89), respectively with $\mathrm{p}<0.0001$. Positive and negative predictive values were $91.8 \%$ (95\%CI: 81.90 - 97.28) (Table 4).

Table 2. PEEK Smartphone Contrast sensitivity and Humphrey visual field analyser $(\mathrm{N}=$ 140).

\begin{tabular}{ccccc}
\hline Test & Measures & Estimate $\%$ & $95 \% \mathrm{CI}$ & Chi-square P value \\
\hline & Sensitivity & 48.91 & $38.3-59.56$ & \\
& Specificity & 54.17 & $39.17-68.63$ & $0.1199>0.05$ \\
Contrast sensitivity & PPV & 67.16 & $54.60-78.15$ & \\
& NPV & 35.62 & $24.75-47.69$ & \\
\hline
\end{tabular}

Table 3. PEEK Smartphone Amsler grid vs. Humphrey visual field analyzer.

\begin{tabular}{ccccc}
\hline Test & Measures & Estimate $\%$ & 95\%CI & Chi-square P value \\
\hline Sensitivity & 33.33 & $20.76-47.92$ & \\
Specificity & 86.52 & $77.63-92.83$ & $7.7785<0.005$ \\
Amsler grid & PPV & 58.62 & $38.94-76.48$ & \\
& NPV & 69.37 & $59.91-77.77$ \\
\hline
\end{tabular}

Table 4. PEEK retina Smartphone f vs. Slit lamp Biomicroscopy.

\begin{tabular}{ccccc}
\hline \multirow{2}{*}{ Test } & Measures & Estimate \% & 95\%CI & Chi-square P value \\
\hline & Sensitivity & 90.32 & $80.12-96.37$ & \\
Specificity & 93.59 & $85.67-97.89$ & $98.9256<0.0001$ \\
PEEK Smartphone & PPV & 91.80 & $81.90-97.28$ \\
\\
NPV & 92.41 & $84.20-97.16$ \\
\hline
\end{tabular}




\section{Discussion}

In the present study PEEK Smartphone for vertical cup to disc ratio measurement showed a promising potential to be used for glaucoma screening in LMICs. Smartphone had a strong agreement (92\%) with Slit lamp biomicroscopy, which is considered a standard screening tool for glaucoma when optic disc parameters were evaluated. Similar to the current studies, several studies, which implemented Smartphone's for glaucoma screening, found strong agreement with standard screening tool [9].

In this study it was also noted that Smartphone agreement (92\%) with Slit lamp biomicroscopy was almost the same with that recorded [10].

The almost same observed agreement between PEEK retinal Smartphone with Slit lamp biomicroscopy in the current study may be due to the quality of image and the almost same sample size.

This means that Smartphone fundus view can be used for glaucoma as sensitivity and specificity and due to its portability and easy to perform the test.

A moderate agreement of 0.51 was found in this study for PEEK Smartphone contrast sensitivity compared with the study done in United States of America, Spain and Turkey, respectively [11] [12] [13]. Those studies presented with the similar results due to refractive error and cataract. These results showed that contrast sensitivity may not be used alone to detect glaucoma because even those patients whereby they do not had glaucoma the contrast sensitivity scored more than $2.5 \%$.

According to the comparison between PEEK Smartphone Amsler grid and Humphrey visual field analyzer (10 - 2), when related with areas of scotoma it was found an agreement of 0.67 which was similar with the study done in United States of America [14]. It was also found that PEEK Smartphone Amsler grid test had lower sensitivity compared with the study done in Switzerland, due to the sample size, severity of the disease and the fact that in the study were associated with wet-related macular generation was studied [15].

It may not be used to screen for glaucoma on the fact that it can miss to diagnose patients at the early stage of the disease.

\section{Limitations}

Some of the pictures from PEEK retina were difficult to evaluate because was not so clear compared with Slit lamp and subjectively were given the results. The auto refractor was giving error for 30 patients.

\section{Conclusion}

PEEK Smartphone fundus view image might be a good tool for screening of optic disc parameters of glaucoma. Its sensitivity and specificity in detecting optic disc damage and its low cost, portability and easy to use make it very more useful in LMICs. 


\section{Acknowledgements}

I would like to express my gratitude to my supervisors, Light-For-The-World, Sia Mbishi, Erasmo Mlay and Kelvin Mbuya to make this study and paper possible. Thank you for guiding me throughout the write-up of the dissertation manuscript.

\section{Conflicts of Interest}

The authors declare no conflicts of interest regarding the publication of this paper.

\section{References}

[1] Pascolini, D. and Mariotti, S.P.M. (2010) Global Estimates of Visual Impairment. British Journal Ophthalmology.

[2] Kyari, F., et al. (2018) The Current Status of Glaucoma and Glaucoma Care in Sub-Saharan Africa. Asia-Pacific Journal of Ophthalmology, 7, 375-386.

[3] Buhrmann, R.R., Quigley, H.A., Barron, Y., West, S.K., Oliva, M.S. and Mmbaga, B.B. (2000) Prevalence of Glaucoma in a Rural East African Population. Investigative Ophthalmology \& Visual Science, 41, 40-48.

[4] Mohammadpour, M., et al. (2017) Smartphones, Tele-Ophthalmology, and Vision 2020. International Journal of Ophthalmology, 10, 1909-1918.

[5] Medeiros, F. and Alencar, L. (2011) The Role of Standard Automated Perimetry and Newer Functional Methods for Glaucoma Diagnosis and Follow-Up. Indian Journal of Ophthalmology, 59, 53. https://doi.org/10.4103/0301-4738.73694

[6] Hajian-Tilaki, K. (2014) Sample Size Estimation in Diagnostic Test Studies of Biomedical Informatics. Journal of Biomedical Informatics, 48, 193-204. https://doi.org/10.1016/j.jbi.2014.02.013

[7] Fellman, R., et al. (2011) Know the New Glaucoma Codes. EyeNet Magazine, 65-66. http://www.aao.org/eyenet/article/know-new-glaucoma-staging-codes?october-201 $\underline{1}$

[8] Susanna Jr., R. and Vessani, R.M. (2009) Staging Glaucoma Patient: Why and How? The Open Ophthalmology Journal, 3, 59-64. https://doi.org/10.2174/1874364100903020059

[9] Russo, A., et al. (2016) Comparison of Smartphone Ophthalmoscopy with Slit-Lamp Biomicroscopy for Grading Vertical Cup-to-Disc Ratio. Journal of Glaucoma, 25, 777-781. https://doi.org/10.1097/IJG.0000000000000499

[10] Boonarpha, N., et al. (2018) Comparison of Optic Disc Images from Two Smartphone-Based Imaging Systems in Glaucoma Patients and Suspects. Ophthalmology Journal, 1, 1-7.

[11] Raza, A.S., Cho, J., de Moraes, C.G.V., et al. (2011) Retinal Ganglion Cell Layer Thickness and Local Visual Field Sensitivity in Glaucoma. Archives of Ophthalmology, 129, 1529-1536. https://doi.org/10.1001/archophthalmol.2011.352

[12] Fuertes, I., et al. (2016) Evaluation of Contrast Sensitivity, Chromatic Vision, and Reading Ability in Patients with Primary Open Angle Glaucoma. Journal of Ophthalmology, 2016, Article ID: 7074016. https://doi.org/10.1155/2016/7074016

[13] Temel, A. (2008) FACT Contrast Sensitivity as a Diagnostic Tool in Glaucoma FACT Contrast Sensitivity in Glaucoma. International Ophthalmology, 28, 407-412. 
https://doi.org/10.1007/s10792-007-9169-Z

[14] Su, D., et al. (2015) Efficacy of the Amsler Grid Test in Evaluating Glaucomatous Central Visual Field Defects. Ophthalmology, 123, 737-743.

[15] Faes, L., et al. (2014) Diagnostic Accuracy of the Amsler Grid and the Preferential Hyperacuity Perimetry in the Screening of Patients with Age-Related Macular Degeneration: Systematic Review and Meta-Analysis. Eye, 28, 788-796.

https://doi.org/10.1038/eye.2014.104 